\title{
Utilisation d'impulsions UV femtosecondes pour l'étude de la relaxation électronique dans $\mathrm{SiO}_{2}$
}

\author{
F. Quéré, S. Guizard, P. Martin, G. Petite, B. Carré, J.-F. Hergott, H. Merdji \\ et $P$. Salières
}

Laboratoire des Solides Irradiés, École Polytechnique, route de Palaiseau, 91128 Palaiseau cedex, France

\begin{abstract}
Résumé : Parmi les techniques permettant d'obtenir des informations sur la structure électronique des solides, la spectroscopie de photoémission occupe une place extrêmement importante. Avec le développement de sources UV et $\mathrm{X}$ impulsionelles, cette technique peut aujourd'bui être utilisée pour étudier les états électroniques excités des solides et leur relaxation, via des expériences de type pompe-sonde. Dans cette perspective, la génération d'harmoniques d'ordres élevés d'un laser femtoseconde intense par focalisation dans un gaz rare apparaît comme l'une des sources de rayonnement UV les plus performantes, puisqu'elle permet d'obtenir des impulsions d'une durée de quelques dizaines de femtosecondes seulement.

Nous avons utilisé cette technique pour réaliser une expérience de photoémission résolue en temps sur un isolant à grande bande interdite, le quartz, avec une résolution temporelle de $100 \mathrm{fs}$. Le faisceau sonde se situait dans l'infrarouge, et les impulsions UV ont été utilisées comme pompe. Des informations sur les différents processus de relaxation d'électrons de quelques dizaines d'eV par rapport au bas de la bande de conduction ont ainsi été obtenues. Nous décrivons le dispositif expérimental, commentons les résultats obtenus, et discutons de futurs développements de ces outils expérimentaux.
\end{abstract}

\section{INTRODUCTION}

Les processus de relaxation dans les solides excités sont une problématique commune à toutes les situations ou se produit une excitation électronique. Un grand nombre de paramètres interviennent dans la dynamique de ces processus. Citons la distribution d'énergie initiale des électrons, qui peut être très différente suivant la source d'ionisation (photons $\gamma, X$ ou UV ou électrons), et la densité d'excitation, très élevée dans le cas d'une impulsion laser femtoseconde intense ou de l'irradiation aux ions lourds ou très faible, lors d'expérience sur rayonnement synchrotron par exemple. Les mécanismes élémentaires de relaxation sont communs à toutes ces expériences : relaxation avec le réseau via le couplage électronsphonons, et relaxations électroniques comme l'ionisation par impact dans les semi-conducteurs ou les isolants. La connaissance de ces processus est donc fondamentale dans de nombreux domaines de la physique des solides et des matériaux.

La technique expérimentale la plus couramment employée pour étudier ces phénomènes est la photoémission résolue en temps, à l'aide d'impulsion laser ultra-brève. Une première impulsion excite le solide, et une seconde impulsion provoque l'ionisation. La mesure de l'énergie cinétique de ces photoélectrons en fonction du retard entre les impulsions pompe et sonde donne une image directe de l'évolution de leur distribution énergétique. Cette technique très puissante a été abondamment utilisée pour étudier les cinétiques de relaxation dans les semi-conducteurs et les métaux. En effet les énergies de photons nécessaires aux excitations électroniques correspondent aux longueurs d'onde d'émission des lasers femtosecondes les plus courants. La génération d'harmoniques d'ordre élevés d'un laser femtoseconde intense dans les gaz rares [1] est une source parfaitement adaptée à cette technique expérimentale puisque les impulsions, naturellement synchrones avec le laser, sont d'une durée égale ou inférieure à celle du laser fondamental. L'énergie des photons qui s'étend jusqu'à plusieurs dizaines d'eV, permet d'étendre considérablement le domaine énergétique accessible, et notamment d'aborder l'étude des matériaux isolants dont la bande interdite -5 à $11 \mathrm{eV}$ - situe les premières excitations électroniques dans l'U.V. Nous avons réalisé une expérience de photoémission du quartz ( $\alpha-\mathrm{SiO}_{2}$ - bande interdite $9 \mathrm{eV}$ ) 
dans laquelle le faisceau "pompe " provoquant l'excitation électronique est l'harmonique 25 d'un laser Titane-Saphir ( $\mathrm{hv}=39 \mathrm{eV}$ ), le faisceau fondamental étant utilisant comme sonde pour ioniser le solide.

\section{DISPOSITIF EXPERIMENTAL}

L'expérience a été réalisée sur la source laser LUCA du centre CEA de Saclay, qui délivre à $800 \mathrm{~nm}$ des impulsions d'une énergie de $100 \mathrm{~mJ}$ et d'une durée de $60 \mathrm{fs}$, avec un taux de répétition de $20 \mathrm{~Hz}$. Une partie du faisceau (environ $10 \mathrm{~mJ}$ ) est utilisée pour générer les harmoniques dans un jet de gaz d'Argon, à partir d'un faisceau annulaire. Le faisceau fondamental résiduel est éliminé à l'aide d'un diaphragme et d'un filtre en aluminium. Les harmoniques sont réfléchies par un miroir revêtu d'un traitement multicouche Bore/Silicium, qui présente un pic de réflectivité autour de $40 \mathrm{eV}$. Le spectre des harmoniques obtenu après l'ensemble miroir - filtre aluminium a été mesuré et présente bien un maximum pour l'harmonique 25 , avec une transmission globale de $5 \%$, soit quelques $10^{6}$ photons par impulsion (pour plus de détails voir [2]). Ce miroir sphérique focalise les harmoniques à incidence quasi normale sur l'échantillon, situé à 3 mètres dans une enceinte à ultra-vide. Une autre partie du faisceau environ $1 \mathrm{~mJ}$, fondamental $(800 \mathrm{~nm})$ ou deuxième harmonique $(400 \mathrm{~nm})$ - constituant la sonde, passe par une ligne à retard et est focalisée sur l'échantillon avec une lentille de focale $2 \mathrm{~m}$. L'énergie des photoélectrons émis à la surface est mesurée à l'aide d'un analyseur hémisphérique multicanal. L'échantillon de quartz est préalablement nettoyé à l'acide fluorhydrique, puis étuvé à $500^{\circ} \mathrm{C}$ à l'air, ce qui permet d'obtenir une surface propre, cristalline et respectant la stœechiométrie [3]. Il est chauffé à $350^{\circ} \mathrm{C}$ pour éviter les problèmes de charge de surface [4]. Nous nous sommes intéressés plus particulièrement aux photoélectrons de plus haute énergie, c'est à dire provenant du haut de la bande de valence. Trois spectres sont reproduits sur la figure 1, obtenus avec le faisceau harmonique seul et avec les deux faisceaux - harmonique et sonde. On remarque un net décalage du spectre d'une énergie correspondant à l'énergie d'un photon sonde $(1.5 \mathrm{eV})$ lorsque les deux impulsions sont synchrones (délai nul) : ces photoélectrons ont donc effectivement absorbés un photon harmonique et un photon sonde. Ce décalage diminue progressivement pour des retards croissants, ce qui montre que les électrons excités par la pompe ont perdu de l'énergie avant d'être ionisés par la sonde. Remarquons également que ce "recul » après $12 \mathrm{ps}$ est moins net pour les électrons de basse énergie(30-31 eV) que pour les électrons de plus haute énergie (33-37 eV), ce qui sera plus visible dans les mesures de cinétiques. L'observation de ce décalage en énergie est le signe que les conditions pour réaliser l'expérience de photoémission résolue en temps proprement dite sont réalisées. On mesure alors le signal de photoélectrons à une énergie donnée en fonction du retard entre les impulsions pompe et sonde. Un premier résultat, illustré par la figure 2,

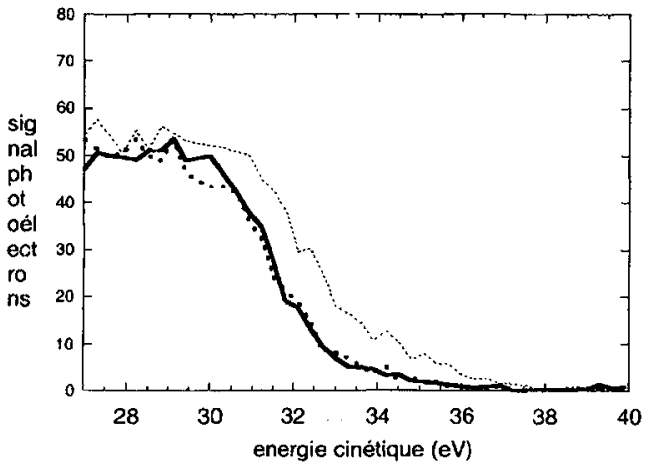

Figure 1 : spectres avec harmonique 25 seule (gras), puis avec $\mathrm{H} 25$ plus sonde, retard $130 \mathrm{fs}$ (pointillés) et -330fs (tirets gras).

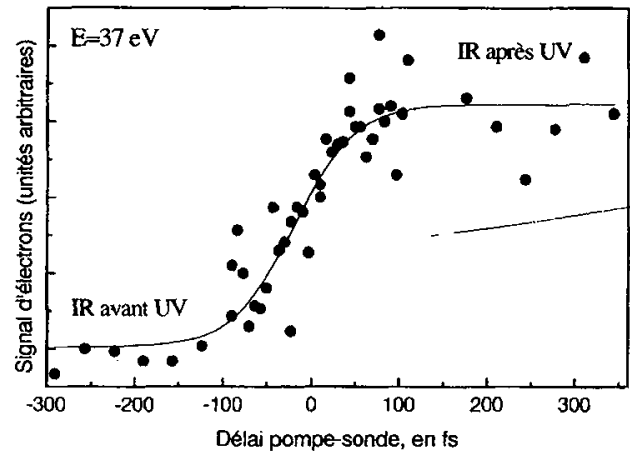

Figure 2 : cinétique aux temps courts. 
permet de mesurer la résolution temporelle accessible par ce type d'expérience. Il s'agit de l'évolution du signal aux temps cours, pour des photoélectrons d'une énergie cinétique de $37 \mathrm{eV}$, qui donne le temps de montée du signal pompe -sonde et donc la fonction de corrélation temporelle entre l'impulsion harmonique et le faisceau Figure1 : fondamental. Dans notre cas la résolution est de l'ordre de 85 fs.

\section{RESULTATS ET DISCUSSION}

Les résultats des cinétiques pour des électrons d'énergies cinétiques $30.8,32 \mathrm{et} 36 \mathrm{eV}$ sont reportés sur les figures 3 et 4 . La brusque augmentation du signal à $t=0$ est suivie d'une diminution d'autant plus lente que l'énergie des électrons est faible. L'interprétation la plus simple de cette observation est que le taux de dépopulation des électrons dans la bande de conduction varie avec l'énergiè. Cependant cette hypothèse implique une décroissance exponentielle du signal, ce qui est incompatible avec les mesures, et
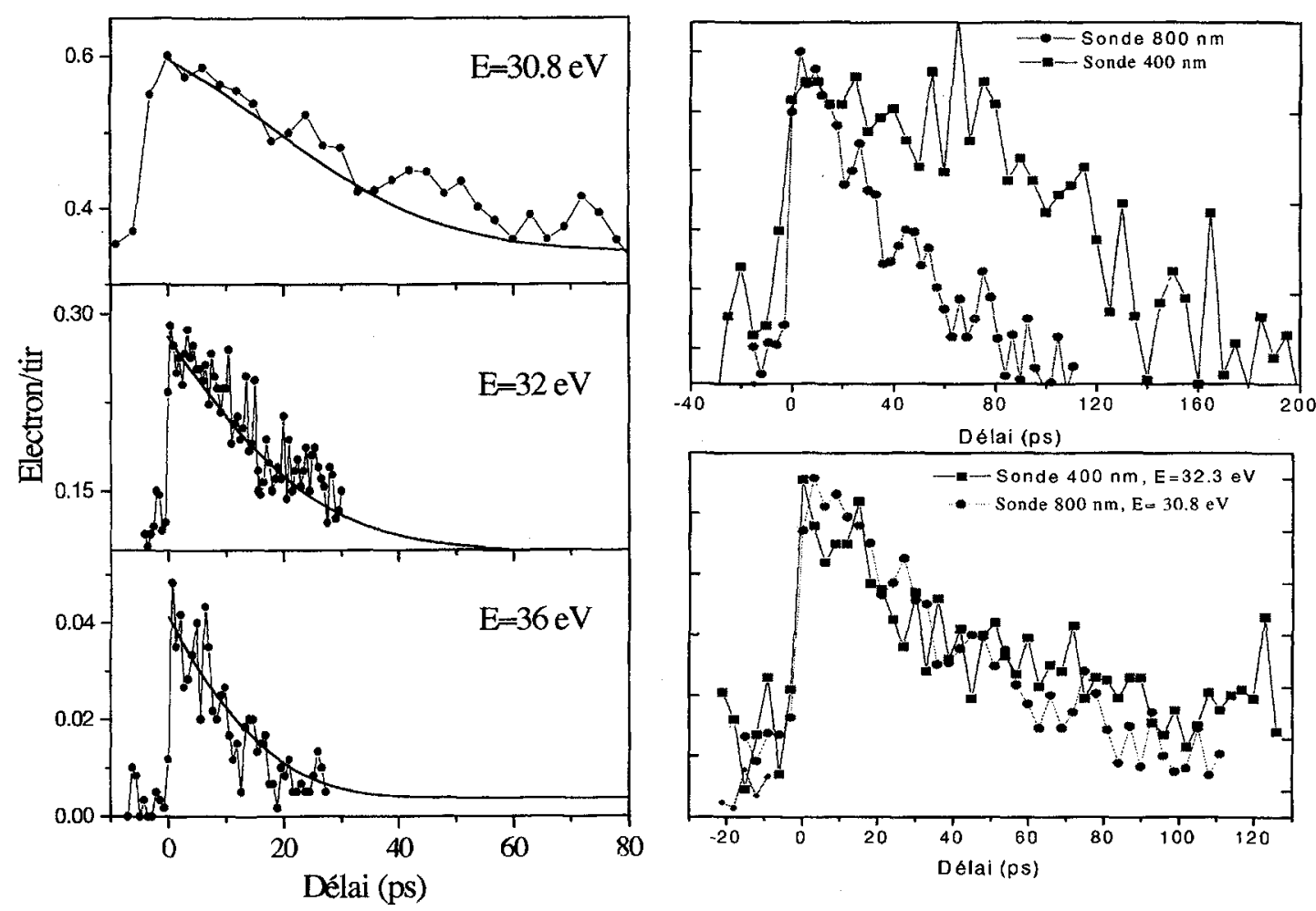

Figure 3 : cinétique aux temps longs, sonde à $800 \mathrm{~nm}$.

Figure 4 : comparaison des cinétiques avec sonde à 400 et $800 \mathrm{~nm}$. Haut : énergie des électrons $30.8 \mathrm{eV}$, Bas : énergie des électrons $32.3 \mathrm{eV}$ (sonde $400 \mathrm{~nm}$ ) et $30.8 \mathrm{eV}$ (sonde $800 \mathrm{~nm}$ ).

de supposer une forte variation du taux d'ionisation par impact dans un gamme d'énergie restreinte e1t située loin du seuil, ce qui semble improbable. Nous interprétons ces résultats de la façon suivante :

- La décroissance du signal est due à une diminution de la population des électrons dans la bande de: conduction qui a pour origine tous les processus induisant des pertes en énergies supérieures à la fenêtre étudiée ici. L'ionisation proprement dite contribue également à cette cinétique. Ces différents; 
processus ne peuvent être discernés dans cette expérience et nous les représenterons par un taux fixe $A$, indépendant de l'énergie dans la gamme de nos mesures.

- Les électrons ayant absorbé un photon UV restent dans la bande de conduction pendant un temps suffisamment long pour perdre une partie de leur énergie avant d'être ionisés par le faisceau sonde.

Dans cette hypothèse, on mesure pour un retard $\Delta t$ entre l'excitation par le photon pompe harmonique et l'ionisation par le photon sonde IR et pour une énergie cinétique $E$ un signal proportionnel à la population d'électrons ayant initialement une énergie $E+\Delta E-h v$, où $h v$ est l'énergie du photon sonde et $\Delta E$ est l'énergie perdue par les électrons pendant l'intervalle de temps $\Delta t$. Ceci explique le fait que les cinétiques sont d'autant plus lentes que l'énergie des photoélectrons est basse. En effet la perte d'énergie $\Delta E$ possible et donc le temps $\Delta t$ nécessaire pour « réaliser » cette perte d'énergie sont d'autant plus important que l'énergie des photoélectrons est basse. Une autre vérification de cette hypothèse est démontrée par les résultats de la figure 4 , où sont comparés les cinétiques mesurées avec des sondes à 400 et $800 \mathrm{~nm}$. Pour sonder les mêmes populations et obtenir les mêmes cinétiques, il faut effectuer des mesures à des énergies d'électrons décalées de la différence d'énergie entre les photons sonde, ce qui est le cas au bas de la figure 4. En revanche des mesures effectuées pour la même énergie de photoélectrons et des énergies de photons sonde différentes ne donnent pas les mêmes cinétiques, comme le montre le haut de la figure 4. Pour quantifier cette hypothèse nous avons modélisé l'évolution de la distribution des électrons dans la bande ce conduction à l'aide d'une série d'équation aux populations : $\frac{d f(E, t)}{d t}=W f(E+\Delta E, t)-(W+A) f(E, t)$, où $f(E, t)$ est la probabilité pour un état d'énergie $E$ d'être occupé à un instant $t$, et $\mathrm{W}$ est pour un électron, la probabilité par unité de temps de perdre une énergie $\Delta E$. Les paramètres $W$ et $A$ sont obtenus en simulant à la fois les spectres de photoélectrons et les cinétiques aux différentes énergies. Les valeurs de ces paramètres permettant de reproduire nos résultats (traits plein sur les figures 2 et 3 ) sont respectivement $70 \mathrm{meV} / \mathrm{ps}$ et $1 / 40 \mathrm{ps}^{-1}$. Ces valeurs sont largement inférieures aux résultats obtenus à l'aide de modèles simples décrivant l'ionisation par impact [5] et les collisions électrons-phonons [6]. Cependant ces modèles sont basés sur des hypothèses (bande parabolique par exemple) qui ne sont sûrement pas valides pour les électrons de grandes énergies cinétiques étudiés ici. La seule expérience, donnant une estimation du taux d'ionisation par impact $A$ de $6 \mathrm{fs}^{-1}$ pour des électrons de $16 \mathrm{eV}$, a été réalisée sur des films minces ( 5 à $20 \AA$ ) de $\mathrm{SiO}_{2}$, dont les propriétés électroniques sont certainement très éloignées de celles d'un monocristal massif [7].

Sans entrer davantage dans la discussion [2], ceci démontre l'intérêt des expériences de photoémission résolue en temps réalisées à l'aide des harmoniques d'ordre élevés. Elles permettent en effet une observation directe des processus de relaxation dans un domaine d'énergie encore totalement inexploré et cette technique peut aisément être appliquée à d'autres matériaux, diélectriques ou non.

\section{Références}

[1] A. L'huillier et al, in Atoms in intense laser fields, edited by M. Gavrila (Academic Press, San Diego, 1992).

[2] Ph. Martin et al., Laser Physics 10, 1 (2000), F. Quéré et al, Phys. Rev. B 61, 9883 (2000).

[3] F. Bart et al., Surface Sciences 306, 342 (1994).

[4] P. Martin, S. Guizard et G. Petite, J. Appl. Phys. 76, 2264 (1994).

[5] B.K. Ridley, Quantum Processes in Semiconductors (Clarendon Press, Oxford, 1993).

[6] H. Fröhlich, Proc. R. Soc. London, Ser A 160, 230 (1937).

[7] E. Cartier et al. Phys rev. B 44, 10689 (1991). 